\title{
Analysis of Supply Chain Information Sharing Mode Based on Big Data
}

\author{
Weijun Lei \\ School of Information Engineering, Xi'an University, No. 1, Science and Technology Sixth Road, \\ Xi'an, China \\ lweijun123@126.com
}

Keywords: Big data, Supply chain, Information sharing, Data storage.

\begin{abstract}
With the wide application of Internet technology, enterprises have formed a large amount of data in the production and operation process. By using big data technology to realize the sharing of supply chain information between enterprises, it plays an important role in improving the operating efficiency of enterprises. This paper first analyzes the concept of regular supply chain information sharing, shared subjects, shared content, and typical sharing patterns. On this basis, research on the application of big data to supply chain information sharing, research on content sharing, information acquisition, different data conversion processing and application methods of supply chain information sharing based on big data, especially based on large The data sharing chain information sharing model is constructed and analyzed, and the theoretical system and application mode of enriching the information sharing of the entire supply chain are discussed.
\end{abstract}

\section{Introduction}

With the development of Internet technology, the generation, processing and application of data have become more convenient. The amount of data in the business process is getting larger and larger, the data information is more transparent, and the conventional enterprise supply chain information sharing method can no longer meet the information demand between enterprises in the Internet environment. The attributes, channels, and methods of information in today's supply chain information sharing are also changing (Fei,Wang and Xinbu, Hu,2015). Using big data and data mining technology can provide more and more useful information for the supply chain of enterprises. It is the important role of big data in the information sharing of enterprise supply chain. This paper studies the sharing of big data and supply chain information as a problem. From the perspective of supply chain information sharing, this paper studies the content, information acquisition, data transformation and application methods of supply chain information sharing in today's big data environment, and builds and analyzes the model based on big data supply chain information sharing. The development of the application first provides some reference from the theoretical model. Through the research of this problem, we can enrich the way of information sharing in supply chain and show the changes brought by big data to supply chain information sharing. Therefore, it is necessary to analyze and study the new challenges faced by supply chain information sharing in the big data environment, and use big data technology and means to 
construct a big data supply chain information sharing model that meets the requirements of the new environment. Provide some references for subsequent practical applications.

\section{Information Sharing}

\subsection{The Concept of Supply Chain Information Sharing}

The so-called supply chain refers to the core enterprise as the center, through the control of the "three streams (information flow, logistics, capital flow)" related to the enterprise, from the procurement of raw materials, the production of intermediate products to the final formation of finished products, and through The sales channel of the enterprise sells the product to the customer, and the overall functional network structure formed by the combination of suppliers, manufacturers, distributors, retailers and end consumers involved in this process (Fei,Wang and Xinbu,Hu,2015).

The so-called supply chain information sharing refers to the use of certain information sharing platforms, channels and technical means (such as database technology) between supply chain node enterprises and their partners to share various information such as demand and orders, and establish relationships between nodes in the supply chain. With the trust outside the supply chain, the systemization of the entire supply chain management and the "win-win" of the node enterprises are finally realized (Zhehui, Zhong, 2010). The essence is to integrate and coordinate the information resources of the entire supply chain through sharing, thereby reducing inventory levels and enterprise costs, improving service response speed and customer satisfaction. The main purpose of information sharing is to avoid the waste of resources and the cost increase caused by information asymmetry, and to achieve optimal distribution of social resources.

In the past, supply chain node members did not pay much attention to information flow, and rarely quantitatively analyzed and evaluated the information flow, resulting in the information flow in the supply chain not being fully utilized, resulting in a slow response of the supply chain to customer demand. Information sharing, especially based on big data supply chain information sharing, is an important solution to this problem (Ning,Dou,2009).

\subsection{The Main Body of Supply Chain Information Sharing}

According to the processes and links of supply chain management, the subjects involved in the traditional supply chain are basically divided into five categories: suppliers, manufacturers, distributors, retailers, and consumers. These subjects are also the subject of information sharing in the supply chain.

\subsection{Contents of Supply Chain Information Sharing}

The content and form of information sharing among the various entities are different due to different industries, operating methods and sales strategies between supply chain nodes. Divided into different content according to different sharing information.

According to the different levels of information, the shared information in the supply chain can be divided into: job information layer, management information layer and decision information layer (Yuan,Meng,2016).

(1) The operation layer information belongs to the micro level of the supply chain operation, and usually refers to some specific information, such as the price, variety, and specific order information of the product, which is reflected in the daily operation of the supply chain.

(2) Management information layer refers to information disseminated between the management layers of each enterprise in the supply chain, such as the company's inventory status, production 
capacity, transportation capacity and lead time.

(3) Decision-making information refers to the information related to the strategic decisionmaking content of the enterprise, including the promotion plan, market forecasting ability, design information of new products, production cost, etc. The sharing of decision-making information is based on rapid response to improve customer satisfaction. For the purpose.

According to the specific content of the information, the shared information in the supply chain mainly includes the following categories (Yun,Zhang and Yuquan,Cui,2008):

(1) Demand information sharing. As marketing strategies have changed from "productionoriented" to "customer-centric", retailers are closest to the end consumer, making sales forecasts based on demand changes and planning and managing inventory.

(2) Sales information sharing. Sales information includes historical sales data and forecasted sales data. The use of information sharing to obtain historical sales data of downstream enterprises, so that upstream member suppliers can obtain the actual demand of the end customers, based on which to make effective production decisions.

(3) Production information sharing. Production information relates to the inventory and sales plans of upstream and downstream companies. It is essential to master the balance between production and sales.

(4) Inventory information sharing. More and more companies are promoting zero inventory in order to save costs. The most important way to share between these companies is to share inventory information.

(5) Order information sharing. In most cases, companies are unaware of the status of their orders and need to share order execution information with other companies in the supply chain for further planning and decision making.

(6) Product information sharing. It mainly includes the sharing of parameter information such as product type, quality and performance. Supply chain nodes mainly establish supply and demand relationships through products.

(7)Transportation informationsharing. Transportation information refers to logistics information. The sharing of transportation information between enterprises can help enterprises to replenish goods quickly and plan the optimal inventory level.

If we only look at the overall efficiency of the supply chain, all the nodes in the supply chain should participate in the process of information sharing, and should share all the information with each other, so that the supply chain operation and management can be transparent. However, in reality, due to the differences between the members of the supply chain, especially the fragmentation of information, coupled with the information flow is not too smooth, affecting the sharing of supply chain information, but with the help of big data technology To provide better conditions for information sharing in the supply chain.

\section{Three Typical Supply Chain Information Sharing Modes}

The information sharing mode is divided according to the direction of information source, the level of information sharing and the control of information sharing.

(1) Point-to-point sharing mode

This mode is the end-to-end information transfer from the supplier to the demand side. In the peer-to-peer mode, the information does not pass through other data centers or platforms.

(2) Information centralized management mode

It is to centralize all the shared information in a common database. In this mode, enterprises can operate according to their respective rights, and can access related information of many of their partners. 
(3) Integrated information sharing model

The integrated information sharing model integrates the peer-to-peer sharing mode and the centralized management mode, emphasizing differentiated information sharing, and generally builds on the information platform.

\section{Conclusions}

Construction of Supply Chain Information Sharing Model Based on Big Data Environment.

\subsection{Changes in Supply Chain Information Sharing in a Big Data Environment}

In recent years, big data has developed rapidly. In the big data environment, the amount of data, the type of data, the generation of data, and the speed of data update are all changing. The emergence of big data has brought about changes in the supply chain. The information sharing method of the supply chain in the big data environment is also different from the past. It is very necessary to study the problem of supply chain information sharing in the data environment.

Mainly reflected in the following three aspects:

1) In the big data environment, the properties of the data have changed. The information content shared between the supply chain entities also changes as the data changes.

2) Information sharing channels and platforms between supply chain enterprises have changed in the big data environment. The supply chain model has been optimized, and the value and effectiveness of supply chain information sharing has also been greatly improved. For example, in “Tao User Evaluation" and "Product Details" in Taobao, the former belongs to information sharing among customers, and the latter belongs to information sharing between suppliers and customers, which is different from previous information sharing methods.

3) The main body of information sharing in the big data environment has changed. In the big data environment, with the popularity of network applications, most consumers can share product evaluation information through various information publishing platforms. However, in the traditional way, most of the evaluation can only be made by means of verbal communication.

\subsection{Data Information Acquisition Based on Big Data Technology}

Today, there is no shortage of data in the supply chain. What is lacking is a suitable model to transform these vast and diversified raw data into actionable information, so as to improve the efficient planning of the supply chain and make critical decisions. Business decision making. In the big data environment, using big data technology to convert a large amount of raw data into information that can be shared is the key and foundation of information sharing. Data acquisition can be divided into three phases: acquiring data, analyzing data, and using data to accomplish business goals (Yuexiang,Zhou,2015).

\subsubsection{Get the data process}

The data acquisition process consists of data source, data separation and cleaning, and data representation. The data sources mainly include production and sales data, release data, inventory data, consumer data, and transportation data, namely GPS and logistics data. Since data usually exists in many forms, only by structured processing of data, data can be effectively used in the process of information sharing in the supply chain, that is, data separation and cleaning. High quality and comprehensive data is a valuable asset in the supply chain, so a suitable data cleaning mechanism can guarantee the quality of big data. 


\subsubsection{Data representation}

In the Internet and big data environment, not only is the data content richer and the data structure is diversified. Therefore, to analyze the application requirements of data, it is also necessary to analyze the expression of big data in the information sharing of enterprise supply chain, and then express the data in an appropriate way to adapt to the supply chain in the big data environment. Information sharing requires data because data plays a key role in supply chain information sharing.

\subsubsection{Analyze the data}

Due to the large amount of data in the big data environment, the data needs to be filtered. Otherwise, a large amount of redundant information will appear. The analysis of the filtered and cleaned data can provide better value for supply chain task planning and forecasting. And services to meet the needs of the supply chain for corporate goals. In general, it is mainly analyzed from input quantities, targets, constraints, outputs, and so on.

\subsubsection{Complete business goals}

The final stage is to accomplish corporate goals through demand forecasting and supply chain planning. In order to approach precise decision making and supply chain optimization goals, the model should support multi-user collaboration so that multiple users can collaborate to create optimal supply chain planning and arrangements, while re-optimizing the business environment as it changes, periodically comparing actual And the results of the plan.

\subsection{Supply Chain Information Sharing Model Based on Big Data Environment}

Using big data technology can mine useful information from massive consumer data. At the same time, in the big data environment, the popularity of cloud computing guarantees the storage problem of massive data, and the capacity limitation becomes no longer obvious. In the following, the supply chain of third-party logistics companies is used as a typical representative to build a new model of supply chain information sharing in the big data environment.

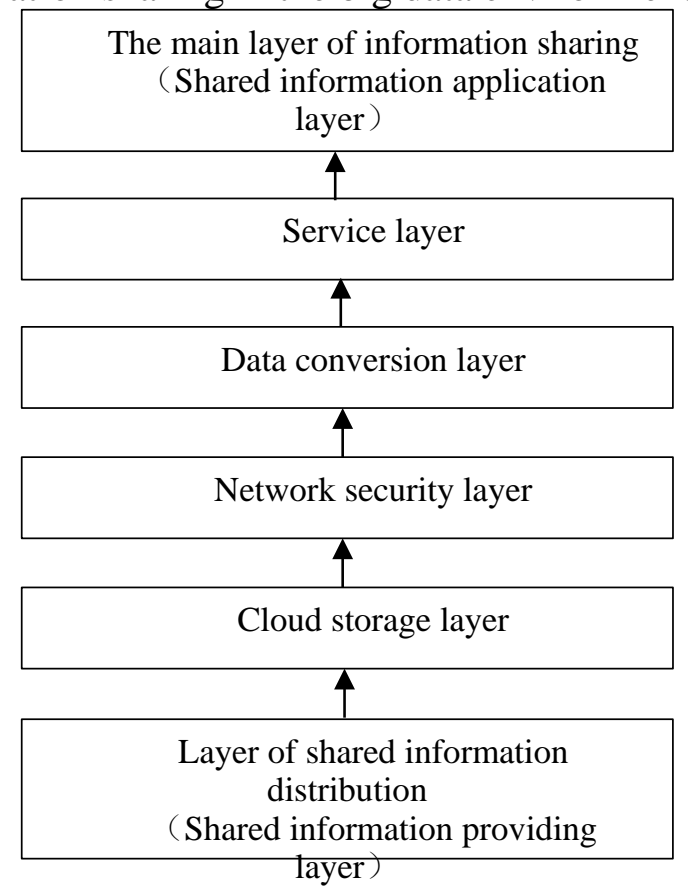

Figure. 1 Supply chain information sharing model in the context of big data environment. 
The supply chain information sharing model is generally divided into three layers: information providing layer, network layer and application layer (Yonggui,He and Wanlei,Zhang,2016). In this paper, the new model of supply chain information sharing in big data environment is divided into six layers: information sharing main layer, service layer, data conversion layer, network security layer, cloud storage layer, and shared information publishing layer. The six layers can be summarized into three components: information sharing participants, shared information content, and big data information sharing platforms. This constructs a model for sharing supply chain information between enterprises based on big data. The model consists of hierarchical functions. The model structure is shown in Figure 1.

The following is a brief description of the functions of the relevant layers in the model.

(1) Information sharing participants

The first layer and the bottom layer are the behaviors of the participants, so they are combined for introduction. They are both providers and publishers of shared information and users of shared information. According to the close cooperation between enterprises, the inter-firm partnerships in the supply chain are divided into three levels, important partners, secondary partners and nonpartners. The three partnerships can be converted to each other, but important partners may also be transformed into secondary or non-partnerships. The types of information shared between them vary according to the level of partnership.

(2) Sharing information content

The publisher of the shared information is the source of the shared information and is the subject that can provide the shared information. The information provided for sharing enters the enterprise's external shared database through the enterprise firewall. The information provided by the participants in the supply chain information sharing is divided into two categories, three levels. The two categories are related to information provided by suppliers and manufacturers, distributors and retailers. Suppliers will send externally shared information from their internal databases to the external shared database through the corporate firewall; consumers will publish their own demand for goods through various e-commerce website comment sections, social networking sites, media and other channels. Comments and other information. These massive, different types of fastchanging giant data are all on the Internet. Since the cooperation relationship between the enterprise and the partner has different grade differences in the information sharing process, it is divided into different levels of shared information forms according to the cooperation level.

(3) Big data information sharing platform

The application of big data in enterprise supply chain information sharing needs to be supported by the system platform. The big data information sharing platform is the core part of the information sharing mode. It includes network security layer, data conversion layer, cloud storage layer, and service layer. The function of each layer will be described below.

Network security layer: mainly includes unified authentication system and monitoring system. The unified authentication system is an access control system based on role assignment related rights. Permissions are set based on roles, and the level of shared information enjoyed by different permissions is different. The user can use the privilege to enjoy the shared information in the shared information database corresponding to the privilege level and obtain the access service. The monitoring system is responsible for the real-time monitoring of the network interface and communication security of the entire big data information sharing platform.

Cloud storage layer: mainly used to store huge amounts of data, it includes three large databases: shared information database, consumer database based on big data analysis, Internet database. (1) The shared information database is an integrated database provided by the information sharing publisher to the external shared information database, and the database is stored on each enterprise's own cloud. This shared information database integrates these databases together; (2) The consumer 
database based on big data analysis is based on the information published by consumers through various channels on the network, using big data technology to mine and analyze this information. And related prediction data; (3) Internet database is a synthetic database that extracts part of the information in the shared information from the shared information database and part of the information extracted from the consumer database based on the big data analysis. Some of the information in the consumer database enters the Internet database, and most of them enter the shared information database and are shared by enterprises in the supply chain to help enterprises make corporate decisions based on consumer feedback. The data between the shared information database and the Interne database can also be passed to each other.

Service layer: mainly provides evaluation services, personalized services, fee-based services, access services, upload and download services. The evaluation system is mainly a system in which each information sharing entity in the supply chain enjoys feedback on its related services after enjoying the information sharing platform; the personalized service and management system is responsible for personalization and setting services related to the information platform interface; It is a charge based on access.

The big data information sharing platform is generally provided by a third-party software provider. The cost of the entire system is collected from the information sharing service enjoyed by each entity sharing information in the big data supply chain; the access service is authenticated by the user and is accessible after payment. Sharing the information authority of the corresponding sharing level in the data, the service can enable the user to access the related information; the uploading and downloading service system is mainly provided to each entity sharing the big data information for publishing and receiving the shared information with lower rank. The system can access the Internet database through the firewall, and can obtain relevant information from the database to provide a download service for the information sharing entity, and can also safely transmit the related data uploaded by the sharing entity to the Internet database.

The new model of supply chain information sharing in the big data environment is mainly a combination of new technologies in the big data environment (such as cloud computing, cloud storage and big data analysis) and traditional integrated shared information models, but because of some special enterprises Its information needs to be kept secret, so it retains the peer-to-peer information sharing model, which is suitable for information sharing needs of enterprises of different scales.

\section{Conclusions}

Based on the analysis of traditional enterprise supply chain information sharing methods, combined with today's Internet and big data technology, this paper studies the application of big data to supply chain information sharing, and considers large databases in enterprise supply chain information sharing. Application is a necessity. According to the characteristics of the data itself, the data source and data conversion process using big data in the supply chain are expounded, especially the analysis of the enterprise supply chain information sharing under the big data environment. The new model and the hierarchical functional structure model are given. The model has been further improved, which provides a useful discussion for big data service and enterprise supply chain information sharing.

\section{Discussion}

With the development and application of big data, Internet of Things, cloud computing and artificial intelligence technology, it will inevitably lead to the transformation of business model. Under the impetus of the Internet and big data, the model of supply chain information sharing 
between enterprises will inevitably change. The information sharing of supply chain based on big data will be developed rapidly. The role of big data in improving information sharing between enterprises will become more and more important, which has important practical significance for improving the quality of business operations. Since the application of big data in enterprise supply chain information sharing is a systematic project, the mode and specific application mode of big data in supply chain information sharing needs further research. Although this article has conducted some discussions in this regard, there are still some shortcomings that need to be refined. In particular, there is a need for in-depth research and improvement in mode hierarchy, hierarchical function settings, and specific applications.

\section{Acknowledgements}

This study is supported by the National College Students' Innovation and Entrepreneurship Training Project (201711080020), Xi'an Social Science Plan Project (16WL12) and Shaanxi Provincial Department of Education 2018 Scientific Research Project (18JK1149).

\section{References}

[1] Fei,Wang and Xinbu,Hu,2015.Review on Several Issues Supply Chain Management, Journal of Human Geography.(3), pp.26-30.

[2] Ning, Dou, 2009.Study on the Value of Information Sharing and Profit Allocation in Three-level Supply Chain, Shandong Normal University Master Degree Dissertation.

[3] Yuan, Meng, 2016.Supply Chain Information Sharing Models and Comparison Analyzing, Journal of Logistics SciTech.(3),pp. 112-114.

[4] Yun,Zhang and Yuquan,Cui,2008. The model of Supply Chains in Part Information Sharing, Journal of Shandong University (Natural Science). (2), pp.77-81+86.

[5] Yuexiang, Zhou, 2015.Research on Supply Chain Information Sharing under Data Environment, Harbin Engineering University Master Degree Dissertation.

[6] Yonggui,He and Wanlei,Zhang,2016.Study on Chemical Supply Chain Information Sharing Mode Based on IOT Technology, Journal of logistics technology.(9),pp.130-135.

[7] Zhehui, Zhong, 2010. On The Model \& Optlmization for Information Sharing Insupply Chain,Southwest Jiaotong University Doctor Degree Dissertation. 